\title{
Dynamical analysis of a new fractional-order predator-prey system with Holling type-III functional
}

\author{
Lihua Dai ${ }^{1,2}$, Junjie Wang ${ }^{2 *}$, Yonggen $\mathrm{Ni}^{2}$ and Bin $\mathrm{Xu}^{2}$
}

\section{"Correspondence:} ynpewjj@126.com 2Department of Mathematics and Statistics, Puer University, Puer, Yunnan 665000, China Full list of author information is available at the end of the article

\section{Springer}

\begin{abstract}
In this paper, we consider a new fractional-order predator-prey model with Holling type-III functional response and stage structure. Based on the Lyapunov stability theory and by constructing a suitable Lyapunov functional, we obtain some sufficient conditions for the existence and uniqueness of positive solutions and the asymptotic stability of the positive equilibrium to the system. Finally, we give some numerical examples to illustrate the feasibility of our results.
\end{abstract}

Keywords: Fractional-order; Predator-prey model; Asymptotic stability; Lyapunov functional

\section{Introduction}

It is well known that the existence and uniqueness of positive solutions for predator-prey models with Holling type-III functional response and stage structure have been widely investigated by many researchers [1-9]. Stage structure is an important natural phenomenon and represents, for example, the division of a population into immature and mature individuals. In [4], the authors studied the global properties of a predator-prey model with nonlinear functional response and stage structure for the predator, and the conditions of the existence and the global stability of the positive steady state were established. In [9], the authors discussed the existence and local stability of equilibrium points, and in order to protect the stability of this kind of systems, they proposed a hybrid control method such that the Hopf bifurcation can be controlled.

Fractional order calculus is the extension of integer order calculus to arbitrary real number order; both appeared almost at the same time. Most of the present works were focused on fractional differential equations, see [10-16] and the references cited therein. The basic theory of fractional calculus can be found in the monographs of Miller and Ross [10]. With the improvement of fractional calculus theory, the fractional differential equations are widely used in various fields, such as physics [17-19], economics [20-22], medicine $[23,24]$, and biology $[25,26]$, etc. Because of the memory and heritage properties of fractional calculus, it is more suitable for describing the population dynamics system than integer calculus. Therefore, it is more in line with the laws of nature and has practical sig-

(c) The Author(s) 2021. This article is licensed under a Creative Commons Attribution 4.0 International License, which permits use sharing, adaptation, distribution and reproduction in any medium or format, as long as you give appropriate credit to the original author(s) and the source, provide a link to the Creative Commons licence, and indicate if changes were made. The images or other third party material in this article are included in the article's Creative Commons licence, unless indicated otherwise in a credit line to the material. If material is not included in the article's Creative Commons licence and your intended use is not permitted by statutory regulation or exceeds the permitted use, you will need to obtain permission directly from the copyright holder. To view a copy of this licence, visit http://creativecommons.org/licenses/by/4.0/. 
nificance to study predator-prey models by using fractional calculus. There are many definitions of fractional derivative and integral, and those of Grünwald-Letnikov, RiemannLiouville, and Caputo are commonly used. To use the Riemann-Liouville definition one must specify the value of a fractional derivative of the unknown solution at the initial value $t=0$, but this can simplify the calculation of the fractional derivative. For complex multiscale analysis, the Riemann-Liouville definition can simplify the calculation process. The shortcomings of the Riemann-Liouville fractional derivative can be compensated by modifying the definition. Therefore, in this paper we will adopt the modified RiemannLiouville-type definition given in [12].

Recently, a fractional predator-prey model to describe the ecosystem was considered in [27], where it performed well on a practical problem. The author considered the fractional predator-prey model with Holling type-II functional response. Later, the research on the dynamics of fractional-order predator-prey model with Holling type-II or Holling type-III functional response has become a hot topic [28-31].

To the best of our knowledge, up to now, few results are available for fractional-order predator-prey systems with Holling type-III functional response and stage structure. Therefore, it is a challenging and important problem in theory and applications.

Motivated by the above discussion, in this paper, we consider the following fractionalorder predator-prey system with Holling type-III functional response and stage structure:

$$
\begin{aligned}
& \frac{d^{\alpha} x_{1}(t)}{d t^{\alpha}}=a x_{2}(t)-r_{1} x_{1}(t)-b x_{1}(t), \\
& \frac{d^{\alpha} x_{2}(t)}{d t^{\alpha}}=b x_{1}(t)-r_{2} x_{2}(t)-b_{1} x_{2}^{2}(t)-\frac{a_{1} x_{2}^{2}(t) y(t)}{1+m x_{2}^{2}(t)}, \\
& \frac{d^{\alpha} y(t)}{d t^{\alpha}}=\frac{a_{2} x_{2}^{2}(t) y(t)}{1+m x_{2}^{2}(t)}-r y(t) \quad(0<\alpha \leq 1),
\end{aligned}
$$

where $x_{1}(t)$ and $x_{2}(t)$ represent the densities of the immature and mature prey at time $t$, respectively; $y(t)$ represents the density of the predator at time $t$; the parameters $a, r_{1}, b$, $r_{2}, b_{1}, a_{1}, m, a_{2}$, and $r$ are positive constants.

Through calculation, it is easy to get that system (1.1) always has a trivial equilibrium $E_{0}(0,0,0)$ and if the condition $a b>r_{2}\left(b+r_{1}\right)$ holds, then (1.1) has a predator-extinction equilibrium $E_{1}\left(x_{1}^{0}, x_{2}^{0}, 0\right)$, where

$$
x_{1}^{0}=\frac{a\left(a b-r_{2}\left(b+r_{1}\right)\right)}{b_{1}\left(b+r_{1}\right)^{2}}, \quad x_{2}^{0}=\frac{a b-r_{2}\left(b+r_{1}\right)}{b_{1}\left(b+r_{1}\right)} .
$$

If the conditions $a b>r_{2}\left(b+r_{1}\right)$ and $\left(a_{2}-m r\right)\left(a b-r_{2}\left(b+r_{1}\right)\right)^{2}>r b_{1}^{2}\left(b+r_{1}\right)^{2}$ hold, then system (1.1) has a unique coexistence equilibrium $E^{*}\left(x_{1}^{*}, x_{2}^{*}, y^{*}\right)$, where

$$
\begin{aligned}
& x_{1}^{*}=\frac{a}{b+r_{1}} x_{2}^{*}, \quad x_{2}^{*}=\sqrt{\frac{r}{a_{2}-m r}}, \\
& y^{*}=\frac{a_{2} x_{2}^{*}}{r a_{1}}\left(\frac{a b}{b+r_{1}}-r_{2}-b_{1} x_{2}^{*}\right) .
\end{aligned}
$$

Our main purpose of this paper is to study the existence and asymptotical stability of equilibria for system (1.1). Especially, we will focus on the considerations that system (1.1) has a unique coexistence equilibrium. 
The main contributions of this paper are listed as follows. Firstly, fractional-order predator-prey system with Holling type-III functional response and stage structure defined by modified fractional derivative is proposed. Secondly, we consider the existence and stability of the equilibrium point of the system, which has important practical significance for the sustainable development of the ecosystem. Finally, examples and numerical simulations are given to verify the effectiveness of the conclusion.

The organization of the rest of this paper is as follows. In Sect. 2, we introduce some good properties on the fractional derivative to study fractional systems. We show that there is a unique nonnegative solution of (1.1) in Sect. 3. In Sect. 4, we use the Lyapunov stability theory [19] of the fractional system to prove that the positive equilibrium is asymptotically stable.

\section{Preliminaries}

In this section, we introduce notations, some basic definitions, and preliminaries that will be used in this paper.

Definition 2.1 ([12]) Let $f: \mathbb{R} \rightarrow \mathbb{R}, x \mapsto f(x)$, be a continuous (but not necessarily differentiable) function, and let $h>0$ be a constant discretization stepsize. Define the forward operator $F W(h)$, i.e., (the symbol := means that the left side is defined by the right one)

$$
F W(h) \cdot f(x):=f(x+h) .
$$

Then the fractional difference of order $\alpha, 0<\alpha<1$, of $f(x)$ is defined by the expression

$$
\Delta^{\alpha} f(x):=(F W-1)^{\alpha} f(x)=\sum_{k=0}^{\infty}(-1)^{k}\left(\begin{array}{l}
\alpha \\
k
\end{array}\right) f(x+(\alpha-k) h),
$$

and its derivative of fractional order is defined by the expression

$$
f^{(\alpha)}(x)=\lim _{h \downarrow 0} \frac{\Delta^{\alpha} f(x)}{h^{\alpha}}, \quad 0<\alpha \leq 1 .
$$

Definition 2.2 ([12], Riemann-Liouville definition revisited) Refer to the function of Definition 2.1. Then its fractional derivative of order $\alpha$ is defined by the expression

$$
f^{(\alpha)}(x)=\frac{1}{\Gamma(-\alpha)} \int_{0}^{x}(x-s)^{-\alpha-1}(f(s)-f(0)) d s, \quad \alpha<0 .
$$

For positive $\alpha$, one will set

$$
f^{(\alpha)}(x)=\left(f^{(\alpha-1)}(x)\right)^{\prime}=\frac{1}{\Gamma(1-\alpha)} \frac{d}{d x} \int_{0}^{x}(x-s)^{-\alpha}(f(s)-f(0)) d s, \quad 0<\alpha<1
$$

and

$$
f^{(\alpha)}(x):=\left(f^{(n)}(x)\right)^{(\alpha-n)}, \quad n \leq \alpha<n+1, n \geq 1
$$

Now, we give some properties of the modified Riemann-Liouville derivative [14] which are used further in this paper: 
(i) $\Gamma(1+\alpha k):=(\alpha k)$ !;

(ii) (Differential relation) $d^{\alpha} f \cong \Gamma(1+\alpha) d f, 0<\alpha \leq 1$.

Lemma 2.1 ([12]) The following equalities hold:

$$
\begin{aligned}
& D^{\alpha} x^{\gamma}=\Gamma(1+\gamma) \Gamma^{-1}(\gamma+1-\alpha) x^{\gamma-\alpha}, \quad \gamma>0, \\
& (u(x) v(x))^{(\alpha)}=u^{\alpha}(x) v(x)+u(x) v^{(\alpha)}(x), \\
& (f[u(x)])^{(\alpha)}=f_{u}^{\prime}(u) u^{(\alpha)}(x) .
\end{aligned}
$$

The solution of the equation

$$
\begin{aligned}
& d^{\alpha} x=f(t) d t^{\alpha}, \quad t \geq 0, \\
& x(0)=x_{0}
\end{aligned}
$$

is defined by the following result:

Lemma 2.2 ([14]) Let $f(t)$ denote a continuous function. Then the solution of $(2.1)$ is defined by the equality

$$
\int_{0}^{t} f(s) d s^{\alpha}=\alpha \int_{0}^{t}(t-s)^{\alpha-1} f(s) d s, \quad 0<\alpha \leq 1 .
$$

In [19], the authors consider the equation

$$
\frac{d^{\alpha} x}{d t^{\alpha}}=f(x)
$$

Lemma 2.3 ([27]) Let $V(x)$ be a $C^{1}$ function satisfying $V(0)=0 ; V(x)>0$. Further,

(i) if $\frac{d^{\alpha} V}{d t^{\alpha}} \leq 0$, then system (2.3) has a stable null solution;

(ii) if $\frac{d^{\alpha} V}{d t^{\alpha}}<0$, then system (2.3) has an asymptotically stable null solution;

(iii) the null solution of system (2.3) is unstable provided $\frac{d^{\alpha} V}{d t^{\alpha}}>0$.

\section{Existence and uniqueness of the nonnegative solution}

Throughout this paper, let $\mathbb{R}_{+}^{3}$ denote the positive cone of $\mathbb{R}^{3}$, namely, $\mathbb{R}_{+}^{3}=\{x=$ $\left.\left(x_{1}, x_{2}, x_{3}\right) \in \mathbb{R}^{3}: x_{i}>0, i=1,2,3\right\}, \overline{\mathbb{R}}_{+}^{3}=\left\{x=\left(x_{1}, x_{2}, x_{3}\right) \in \mathbb{R}^{3}: x_{i} \geq 0, i=1,2,3\right\}$.

Theorem 3.1 For any initial value $\left(x_{1}(0), x_{2}(0), y(0)\right) \in \mathbb{R}_{+}^{3}$, system $(1.1)$ has a unique global positive solution $\left(x_{1}(t), x_{2}(t), y(t)\right) \in \mathbb{R}_{+}^{3}$ for $t \geq 0$.

Proof Since the right-hand side coefficients of the system (1.1) are locally Lipschitz continuous, referring to the proof of Theorem 4 of [27], we omit the proof of existence of a local solution in this paper. Hence, for any given initial value $\left(x_{1}(0), x_{2}(0), y(0)\right) \in \mathbb{R}_{+}^{3}$, there is a unique maximal local solution $\left(x_{1}(t), x_{2}(t), y(t)\right)$ on $t \in\left[0, \tau_{e}\right)$, where $\tau_{e}$ is the explosion time. To show this solution is global, we need to show that $\tau_{e}=\infty$. Let $k_{0} \geq 1$ be sufficiently large so that $x_{1}(0), x_{2}(0)$ and $y(0)$ all lie within the interval $\left[\frac{1}{k_{0}}, k_{0}\right]$. For each integer $k>k_{0}$, define the stopping time by

$$
\tau_{k}=\inf \left\{t \in\left[0, \tau_{e}\right): \min \left\{x_{1}(t), x_{2}(t), y(t)\right\} \leq \frac{1}{k} \text { or } \max \left\{x_{1}(t), x_{2}(t), y(t)\right\} \geq k\right\} \text {, }
$$


where, throughout this paper, we set inf $\emptyset=\infty$ ( $\emptyset$ denotes the empty set). Obviously, $\tau_{k}$ is increasing as $k \rightarrow \infty$. Set $\tau_{\infty}=\lim _{k \rightarrow \infty} \tau_{k}$, where $\tau_{\infty}<\tau_{e}$. If we can show that $\tau_{\infty}=\infty$, then $\tau_{e}=\infty$ and $\left(x_{1}(t), x_{2}(t), y(t)\right) \in \mathbb{R}_{+}^{3}$ for all $t \geq 0$. Namely, to complete the proof, it is sufficient to show that $\tau_{\infty}=\infty$. If this statement is false, then there is a constant $T>0$ such that

$$
\tau_{\infty} \leq T
$$

Hence, there is an integer $k_{1}>k_{0}$ such that

$$
\tau_{k} \leq T, \quad \forall k \geq k_{1}
$$

where $k_{0}$ satisfies $\frac{1}{k_{0}}<x_{i}(0)<k_{0}, i=1,2$, and $\frac{1}{k_{0}}<y(0)<k_{0}$.

Define a $C^{2}$-function $V: \mathbb{R}_{+}^{3} \rightarrow \overline{\mathbb{R}}_{+}$by

$$
\begin{aligned}
V\left(x_{1}, x_{2}, y\right)= & \left(\sqrt{x_{1}}-1-\frac{1}{2} \log x_{1}\right)+\left(\sqrt{x_{2}}-1-\frac{1}{2} \log x_{2}\right) \\
& +\left(\sqrt{y}-1-\frac{1}{2} \log y\right)
\end{aligned}
$$

the nonnegativity of this function can be seen from $u-1-\log u \geq 0, \forall u$.

Using the chain rule, we get

$$
\begin{aligned}
\frac{d^{\alpha} V}{d t^{\alpha}}= & \frac{\partial V}{\partial x_{1}} \frac{d^{\alpha} x_{1}}{d t^{\alpha}}+\frac{\partial V}{\partial x_{2}} \frac{d^{\alpha} x_{2}}{d t^{\alpha}}+\frac{\partial V}{\partial y} \frac{d^{\alpha} y}{d t^{\alpha}} \\
= & \frac{1}{2}\left(\frac{1}{\sqrt{x_{1}}}-\frac{1}{x_{1}}\right)\left(a x_{2}-r_{1} x_{1}-b x_{1}\right) \\
& +\frac{1}{2}\left(\frac{1}{\sqrt{x_{2}}}-\frac{1}{x_{2}}\right)\left(b x_{1}-r_{2} x_{2}-b_{1} x_{2}^{2}-\frac{a_{1} x_{2}^{2} y}{1+m x_{2}^{2}}\right) \\
& +\frac{1}{2}\left(\frac{1}{\sqrt{y}}-\frac{1}{y}\right)\left(\frac{a_{2} x_{2}^{2} y}{1+m x_{2}^{2}}-r y\right) \\
= & \frac{1}{2}\left(\sqrt{x_{1}}-1\right)\left(-r_{1}-b\right)+\frac{1}{2}\left(\sqrt{x_{2}}-1\right)\left(-r_{2}-b_{1} x_{2}-\frac{a_{1} x_{2} y}{1+m x_{2}^{2}}\right) \\
& +\frac{1}{2}(\sqrt{y}-1)\left(\frac{a_{2} x_{2}^{2}}{1+m x_{2}^{2}}-r\right)+\frac{1}{2}\left(\frac{1}{\sqrt{x_{1}}}-\frac{1}{x_{1}}\right) a x_{2} \\
& +\frac{1}{2}\left(\frac{1}{\sqrt{x_{2}}}-\frac{1}{x_{2}}\right) b x_{1} .
\end{aligned}
$$

Now, we pay attention to the term $\frac{1}{2}\left(\frac{1}{\sqrt{x_{1}}}-\frac{1}{x_{1}}\right) a x_{2}$. If $x_{1}<1$, then $\frac{1}{2}\left(\frac{1}{\sqrt{x_{1}}}-\frac{1}{x_{1}}\right) a x_{2}<0$, hence, this term can be omitted from the right-hand side of the inequality. If $x_{1}>1$, then $\frac{1}{2}\left(\frac{1}{\sqrt{x_{1}}}-\right.$ $\left.\frac{1}{x_{1}}\right) a x_{2}<\frac{1}{2}\left(\sqrt{x_{1}}-1\right) a x_{2}$. Similarly, we have $\frac{1}{2}\left(\frac{1}{\sqrt{x_{2}}}-\frac{1}{x_{2}}\right) b x_{1}<\frac{1}{2}\left(\sqrt{x_{2}}-1\right) b x_{1}$. So we get the following inequality:

$$
\begin{aligned}
\frac{d^{\alpha} V}{d t^{\alpha}} \leq & \frac{1}{2}\left[r_{1}+b-\sqrt{x_{1}}\left(r_{1}+b\right)\right]+\frac{a}{2} x_{2}\left(\sqrt{x_{1}}-1\right)+\frac{b}{2} x_{1}\left(\sqrt{x_{2}}-1\right) \\
& +\frac{1}{2}\left[-b_{1} \sqrt[3]{x_{2}^{2}}+b_{1} x_{2}-r_{2} \sqrt{x_{2}}+r_{2}-\frac{a_{1}}{m}\right]+\frac{1}{2}\left[r+\left(\frac{a_{2}}{m}-r\right) \sqrt{y}\right]
\end{aligned}
$$




$$
\begin{aligned}
\leq & \frac{1}{2}\left[\frac{b}{2} x_{1}^{2}-b x_{1}-\sqrt{x_{1}}\left(r_{1}+b\right)+r_{1}+b\right]+\frac{1}{2}\left[r+\left(\frac{a_{2}}{m}-r\right) \sqrt{y}\right] \\
& +\frac{1}{2}\left[\frac{a}{2} x_{2}^{2}-b_{1} \sqrt[3]{x_{2}^{2}}+\left(b_{1}-a\right) x_{2}-r_{2} \sqrt{x_{2}}+r_{2}-\frac{a_{1}}{m}\right] \\
\leq & M
\end{aligned}
$$

where $M$ is a positive constant. Then $d^{\alpha} V \leq M d t^{\alpha}$. Therefore, on the one hand,

$$
\int_{0}^{\tau_{k} \wedge T} d^{\alpha} V\left(x_{1}(t), x_{2}(t), y(t)\right) \leq \int_{0}^{\tau_{k} \wedge T} M d t^{\alpha} \leq M \int_{0}^{T} d t^{\alpha}
$$

Using equality (2.2), we get

$$
\int_{0}^{T} d t^{\alpha}=\alpha \int_{0}^{T}(T-t)^{\alpha-1} d t, \quad 0<\alpha \leq 1,
$$

which implies that

$$
\int_{0}^{\tau_{k} \wedge T} d^{\alpha} V\left(x_{1}(t), x_{2}(t), y(t)\right) \leq M \alpha \int_{0}^{T}(T-t)^{\alpha-1} d t=M T^{\alpha}
$$

On the other hand, using the differential relation $d^{\alpha} f=\Gamma(1+\alpha) d f$, we have

$$
\begin{aligned}
\int_{0}^{\tau_{k} \wedge T} d^{\alpha} V & =\int_{0}^{\tau_{k} \wedge T} \Gamma(1+\alpha) d V \\
& =\Gamma(1+\alpha)\left[V\left(x_{1}\left(\tau_{k} \wedge T\right), x_{2}\left(\tau_{k} \wedge T\right), y\left(\tau_{k} \wedge T\right)\right)-V\left(x_{1}(0), x_{2}(0), y(0)\right)\right] .
\end{aligned}
$$

Therefore

$$
V\left(x_{1}\left(\tau_{k} \wedge T\right), x_{2}\left(\tau_{k} \wedge T\right), y\left(\tau_{k} \wedge T\right)\right) \leq V\left(x_{1}(0), x_{2}(0), y(0)\right)+\frac{M T^{\alpha}}{\Gamma(1+\alpha)} .
$$

Set $\Omega_{k}=\left\{\tau_{k} \leq T\right\}$ for $k \geq k_{1}$. Note that if at least one of $x_{1}\left(\tau_{k}\right), x_{2}\left(\tau_{k}\right)$ and $y\left(\tau_{k}\right)$ equals either $k$ or $\frac{1}{k}$, we know that $V\left(x_{1}\left(\tau_{k} \wedge T\right), x_{2}\left(\tau_{k} \wedge T\right), y\left(\tau_{k} \wedge T\right)\right)$ is no less than $\min \{(\sqrt{k}-$ $\left.\left.1-\frac{1}{2} \ln k\right),\left(\frac{1}{\sqrt{k}}-1-\frac{1}{2} \ln \frac{1}{k}\right)\right\}$. It then follows from (3.4) that

$$
\begin{aligned}
& \min \left\{\left(\sqrt{k}-1-\frac{1}{2} \ln k\right),\left(\frac{1}{\sqrt{k}}-1-\frac{1}{2} \ln \frac{1}{k}\right)\right\} \\
& \leq V\left(x_{1}(0), x_{2}(0), y(0)\right)+\frac{M T^{\alpha}}{\Gamma(1+\alpha)} .
\end{aligned}
$$

Letting $k \rightarrow \infty$ leads to the contradiction

$$
\infty>V\left(x_{1}(0), x_{2}(0), y(0)\right)+\frac{M T^{\alpha}}{\Gamma(1+\alpha)}=\infty \text {. }
$$

This contradiction shows that $\tau_{\infty}=\infty$, which completes the proof. 


\section{The stability of the solution}

In this section, by constructing an appropriate Lyapunov functional, we shall study the stability of the solution of system (1.1).

Theorem 4.1 If $\left(a_{2}-m r\right)\left(a b-r_{2}\left(b+r_{1}\right)\right)^{2}>r b_{1}^{2}\left(b+r_{1}\right)^{2}$, then the positive equilibrium $\left(x_{1}^{*}, x_{2}^{*}, y^{*}\right)$ of system (1.1) is asymptotically stable, i.e., for any initial value $\left(x_{1}(0), x_{2}(0)\right.$, $y(0)) \in \mathbb{R}_{+}^{3}$, the solution of system (1.1) has the property that $\lim _{t \rightarrow+\infty} x_{1}(t)=x_{1}^{*}$, $\lim _{t \rightarrow+\infty} x_{2}(t)=x_{2}^{*}, \lim _{t \rightarrow+\infty} y(t)=y^{*}$.

Proof Since $\left(a_{2}-m r\right)\left(a b-r_{2}\left(b+r_{1}\right)\right)^{2}>r b_{1}^{2}\left(b+r_{1}\right)^{2}$, then there is a positive equilibrium $\left(x_{1}^{*}, x_{2}^{*}, y^{*}\right)$ of system $(1.1)$, and

$$
\begin{aligned}
& r_{1}=\frac{a x_{2}^{*}-b x_{1}^{*}}{x_{1}^{*}}, \quad r_{2}=\frac{b x_{1}^{*}}{x_{2}^{*}}-\frac{a_{1} x_{2}^{*} y^{*}}{1+m\left(x_{2}^{*}\right)^{2}}-b_{1} x_{2}^{*}, \\
& r=\frac{a_{2}\left(x_{2}^{*}\right)^{2}}{1+m\left(x_{2}^{*}\right)^{2}} .
\end{aligned}
$$

System (1.1) can be rewritten as

$$
\begin{aligned}
\frac{d^{\alpha} x_{1}}{d t^{\alpha}}= & \frac{a}{x_{1}^{*}}\left[x_{1}\left(x_{2}-x_{2}^{*}\right)-x_{2}\left(x_{1}-x_{1}^{*}\right)\right], \\
\frac{d^{\alpha} x_{2}}{d t^{\alpha}}= & \frac{b}{x_{2}^{*}}\left[x_{2}\left(x_{1}-x_{1}^{*}\right)-x_{1}\left(x_{2}-x_{2}^{*}\right)\right]-b_{1} x_{2}\left(x_{2}-x_{2}^{*}\right) \\
& +a_{1} x_{2} \frac{m x_{2} x_{2}^{*} y^{*}\left(x_{2}-x_{2}^{*}\right)-x_{2}\left(1+m\left(x_{2}^{*}\right)^{2}\right)\left(y-y^{*}\right)-y^{*}\left(x_{2}-x_{2}^{*}\right)}{\left(1+m x_{2}^{2}\right)\left(1+m\left(x_{2}^{*}\right)^{2}\right)} \\
\frac{d^{\alpha} y}{d t^{\alpha}}= & a_{2} y \frac{\left(x_{2}+x_{2}^{*}\right)\left(x_{2}-x_{2}^{*}\right)}{\left(1+m x_{2}^{2}\right)\left(1+m\left(x_{2}^{*}\right)^{2}\right)} \quad(0<\alpha \leq 1) .
\end{aligned}
$$

Define a Lyapunov function

$$
\begin{aligned}
V\left(x_{1}, x_{2}, y\right)= & c_{1}\left(x_{1}-x_{1}^{*}-x_{1}^{*} \ln \left(\frac{x_{1}}{x_{1}^{*}}\right)\right) \\
& +c_{2}\left(x_{2}-x_{2}^{*}-x_{2}^{*} \ln \left(\frac{x_{2}}{x_{2}^{*}}\right)\right) \\
& +c_{3}\left(y-y^{*}-y^{*} \ln \left(\frac{y}{y^{*}}\right)\right),
\end{aligned}
$$

where $c_{i}(i=1,2,3)$ are positive numbers to be determined; the nonnegativity of this function can be seen from the property that $\lim _{t \rightarrow+\infty} x_{1}(t)=x_{1}^{*}, \lim _{t \rightarrow+\infty} x_{2}(t)=x_{2}^{*}$, $\lim _{t \rightarrow+\infty} y(t)=y^{*}$.

Then using the chain rule, we obtain

$$
\begin{aligned}
\frac{d^{\alpha} V}{d t^{\alpha}}= & \frac{\partial V}{\partial x_{1}} \frac{d^{\alpha} x_{1}}{d t^{\alpha}}+\frac{\partial V}{\partial x_{2}} \frac{d^{\alpha} x_{2}}{d t^{\alpha}}+\frac{\partial V}{\partial y} \frac{d^{\alpha} y}{d t^{\alpha}} \\
= & c_{1}\left(1-\frac{x_{1}^{*}}{x_{1}}\right) \frac{a}{x_{1}^{*}}\left[x_{1}\left(x_{2}-x_{2}^{*}\right)-x_{2}\left(x_{1}-x_{1}^{*}\right)\right] \\
& +c_{2}\left(1-\frac{x_{2}^{*}}{x_{2}}\right) \frac{b}{x_{2}^{*}}\left[x_{2}\left(x_{1}-x_{1}^{*}\right)-x_{1}\left(x_{2}-x_{2}^{*}\right)\right]
\end{aligned}
$$




$$
\begin{aligned}
& +c_{2}\left(1-\frac{x_{2}^{*}}{x_{2}}\right) a_{1} x_{2} \frac{m x_{2} x_{2}^{*} y^{*}\left(x_{2}-x_{2}^{*}\right)-x_{2}\left(1+m\left(x_{2}^{*}\right)^{2}\right)\left(y-y^{*}\right)-y^{*}\left(x_{2}-x_{2}^{*}\right)}{\left(1+m x_{2}^{2}\right)\left(1+m\left(x_{2}^{*}\right)^{2}\right)} \\
& -c_{2}\left(1-\frac{x_{2}^{*}}{x_{2}}\right) b_{1} x_{2}\left(x_{2}-x_{2}^{*}\right)+c_{3}\left(1-\frac{y^{*}}{y}\right) a_{2} y \frac{\left(x_{2}+x_{2}^{*}\right)\left(x_{2}-x_{2}^{*}\right)}{\left(1+m x_{2}^{2}\right)\left(1+m\left(x_{2}^{*}\right)^{2}\right)} \\
& =c_{1}\left(x_{1}-x_{1}^{*}\right) \frac{a}{x_{1}^{*}}\left[\left(x_{2}-x_{2}^{*}\right)-\frac{x_{2}}{x_{1}}\left(x_{1}-x_{1}^{*}\right)\right] \\
& +c_{2}\left(x_{2}-x_{2}^{*}\right) \frac{b}{x_{2}^{*}}\left[\left(x_{1}-x_{1}^{*}\right)-\frac{x_{1}}{x_{2}}\left(x_{2}-x_{2}^{*}\right)\right] \\
& +a_{1} c_{2}\left(x_{2}-x_{2}^{*}\right) \frac{m x_{2} x_{2}^{*} y^{*}\left(x_{2}-x_{2}^{*}\right)-x_{2}\left(1+m\left(x_{2}^{*}\right)^{2}\right)\left(y-y^{*}\right)-y^{*}\left(x_{2}-x_{2}^{*}\right)}{\left(1+m x_{2}^{2}\right)\left(1+m\left(x_{2}^{*}\right)^{2}\right)} \\
& -c_{2} b_{1}\left(x_{2}-x_{2}^{*}\right)^{2}+a_{2} c_{3}\left(y-y^{*}\right) \frac{\left(x_{2}+x_{2}^{*}\right)\left(x_{2}-x_{2}^{*}\right)}{\left(1+m x_{2}^{2}\right)\left(1+m\left(x_{2}^{*}\right)^{2}\right)} .
\end{aligned}
$$

Set $c_{1}=\frac{x_{1}^{*}}{a}, c_{2}=\frac{x_{2}^{*}}{b}, c_{3}=\frac{a_{1} 1_{2}^{*} y^{*}\left(1+m\left(x_{2}^{*}\right)^{2}\right)}{b a_{2}\left(x_{2}+x_{2}^{*}\right)}$. Then we have

$$
\begin{aligned}
\frac{d^{\alpha} V}{d t^{\alpha}}= & \frac{x_{1}^{*}}{a}\left(x_{1}-x_{1}^{*}\right) \frac{a}{x_{1}^{*}}\left[\left(x_{2}-x_{2}^{*}\right)-\frac{x_{2}}{x_{1}}\left(x_{1}-x_{1}^{*}\right)\right] \\
& +\frac{x_{2}^{*}}{b}\left(x_{2}-x_{2}^{*}\right) \frac{b}{x_{2}^{*}}\left[\left(x_{1}-x_{1}^{*}\right)-\frac{x_{1}}{x_{2}}\left(x_{2}-x_{2}^{*}\right)\right] \\
& +a_{1} \frac{x_{2}^{*}}{b}\left(x_{2}-x_{2}^{*}\right) \frac{m x_{2} x_{2}^{*} y^{*}\left(x_{2}-x_{2}^{*}\right)-x_{2}\left(1+m\left(x_{2}^{*}\right)^{2}\right)\left(y-y^{*}\right)-y^{*}\left(x_{2}-x_{2}^{*}\right)}{\left(1+m x_{2}^{2}\right)\left(1+m\left(x_{2}^{*}\right)^{2}\right)} \\
& -\frac{x_{2}^{*}}{b} b_{1}\left(x_{2}-x_{2}^{*}\right)^{2}+a_{2} \frac{a_{1} x_{2}^{*} y^{*}\left(1+m\left(x_{2}^{*}\right)^{2}\right)}{b a_{2}\left(x_{2}+x_{2}^{*}\right)}\left(y-y^{*}\right) \frac{\left(x_{2}+x_{2}^{*}\right)\left(x_{2}-x_{2}^{*}\right)}{\left(1+m x_{2}^{2}\right)\left(1+m\left(x_{2}^{*}\right)^{2}\right)} \\
= & \left.-\frac{x_{2}}{x_{1}}\left(x_{1}-x_{1}^{*}\right)^{2}+2\left(x_{1}-x_{1}^{*}\right)\left(x_{2}-x_{2}^{*}\right)-\frac{x_{1}}{x_{2}}\left(x_{2}-x_{2}^{*}\right)^{2}\right] \\
& -\frac{x_{2}^{*}}{b}\left[b_{1}-\frac{a_{1} m x_{2} x_{2}^{*} y^{*}-a_{1} y^{*}}{\left(1+m x_{2}^{2}\right)\left(1+m\left(x_{2}^{*}\right)^{2}\right)}\right]\left(x_{2}-x_{2}^{*}\right)^{2} \\
& +\frac{a_{1} x_{2} x_{2}^{*} y^{*}\left(1+m\left(x_{2}^{*}\right)^{2}\right)-a_{1} x_{2} x_{2}^{*} y^{*}\left(1+m\left(x_{2}^{*}\right)^{2}\right)}{b\left(1+m x_{2}^{2}\right)\left(1+m\left(x_{2}^{*}\right)^{2}\right)}\left(x_{2}-x_{2}^{*}\right)\left(y-y^{*}\right) \\
\leq & -\left[\sqrt{\frac{x_{2}}{x_{1}}}\left(x_{1}-x_{1}^{*}\right)-\sqrt{\frac{x_{1}}{x_{2}}}\left(x_{2}-x_{2}^{*}\right)\right]^{2} \\
& -\frac{x_{2}^{*}}{b}\left[b_{1}-\frac{a_{1} m x_{2}^{*} y^{*}-a_{1} y^{*}}{\left(1+m\left(x_{2}^{*}\right)^{2}\right)}\right]\left(x_{2}-x_{2}^{*}\right)^{2} .
\end{aligned}
$$

Then, from Lemma 2.3 and the condition of Theorem 4.1, the above implies $\frac{d^{\alpha} V}{d t^{\alpha}}<0$. Thus system (1.1) is asymptotically stable. The proof is complete.

\section{Numerical examples}

In this section, we give numerical examples to illustrate the theoretical results above. The simulation results are based on Adams-Bashforth-Moulton predictor-corrector scheme [32] and step-length $\Delta t=0.01$. 
Example 5.1 Consider the following fractional order predator-prey system:

$$
\begin{aligned}
& \frac{d^{\alpha} x_{1}(t)}{d t^{\alpha}}=a x_{2}(t)-r_{1} x_{1}(t)-b x_{1}(t), \\
& \frac{d^{\alpha} x_{2}(t)}{d t^{\alpha}}=b x_{1}(t)-r_{2} x_{2}(t)-b_{1} x_{2}^{2}(t)-\frac{a_{1} x_{2}^{2}(t) y(t)}{1+m x_{2}^{2}(t)}, \\
& \frac{d^{\alpha} y(t)}{d t^{\alpha}}=\frac{a_{2} x_{2}^{2}(t) y(t)}{1+m x_{2}^{2}(t)}-r y(t) \quad(0<\alpha \leq 1),
\end{aligned}
$$

and let $\alpha=0.95, a=6, a_{1}=\frac{2}{5}, a_{2}=3, b=2, b_{1}=4, r_{1}=r_{2}=r=\frac{1}{2}, m=2$. It is easy to show that

$$
\left(a_{2}-m r\right)\left(a b-r_{2}\left(b+r_{1}\right)\right)^{2}-r b_{1}^{2}\left(b+r_{1}\right)^{2}=181.125>0 \text {. }
$$

Hence, system (5.1) has a unique coexistence equilibrium

$$
\begin{aligned}
& x_{1}^{*}=\frac{a}{b+r_{1}} x_{2}^{*}=\frac{6}{5}, \quad x_{2}^{*}=\sqrt{\frac{r}{a_{2}-m r}}=\frac{1}{2}, \\
& y^{*}=\frac{a_{2} x_{2}^{*}}{r a_{1}}\left(\frac{a b}{b+r_{1}}-r_{2}-b_{1} x_{2}^{*}\right)=\frac{69}{4},
\end{aligned}
$$

which is asymptotically stable. The results are verified by the numerical simulations in Figs. 1-3.

Example 5.2 Consider the following fractional order predator-prey system:

$$
\begin{aligned}
& \frac{d^{\alpha} x_{1}(t)}{d t^{\alpha}}=a x_{2}(t)-r_{1} x_{1}(t)-b x_{1}(t) \\
& \frac{d^{\alpha} x_{2}(t)}{d t^{\alpha}}=b x_{1}(t)-r_{2} x_{2}(t)-b_{1} x_{2}^{2}(t)-\frac{a_{1} x_{2}^{2}(t) y(t)}{1+m x_{2}^{2}(t)} \\
& \frac{d^{\alpha} y(t)}{d t^{\alpha}}=\frac{a_{2} x_{2}^{2}(t) y(t)}{1+m x_{2}^{2}(t)}-r y(t) \quad(0<\alpha \leq 1)
\end{aligned}
$$

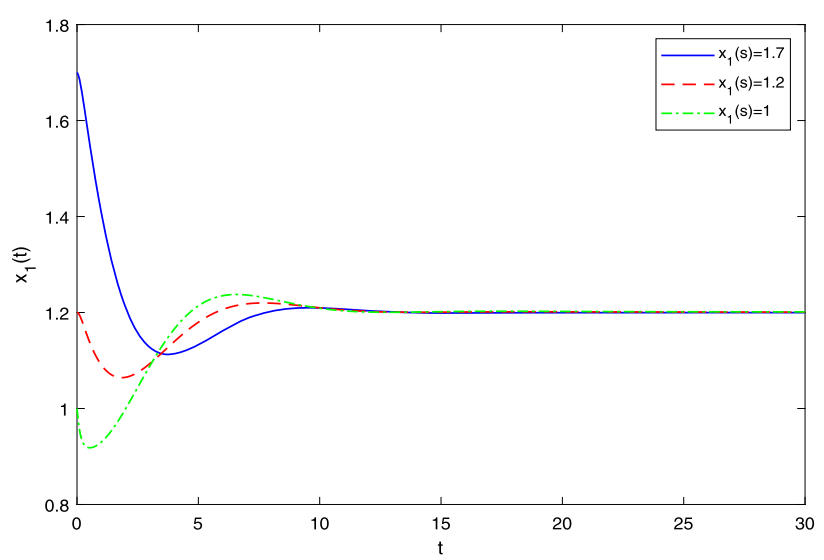

Figure 1 Time responses of state variable $x_{1}(t)$ with $\alpha=0.95$ 


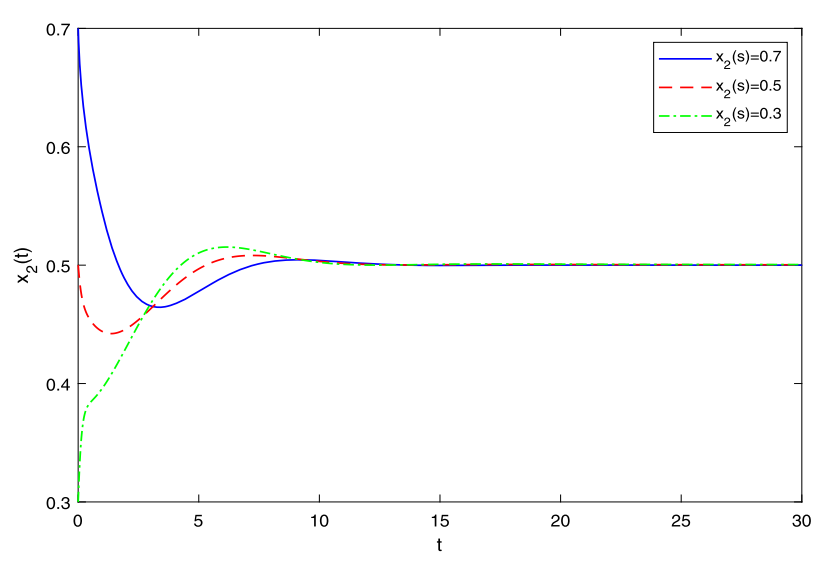

Figure 2 Time responses of state variable $x_{2}(t)$ with $\alpha=0.95$

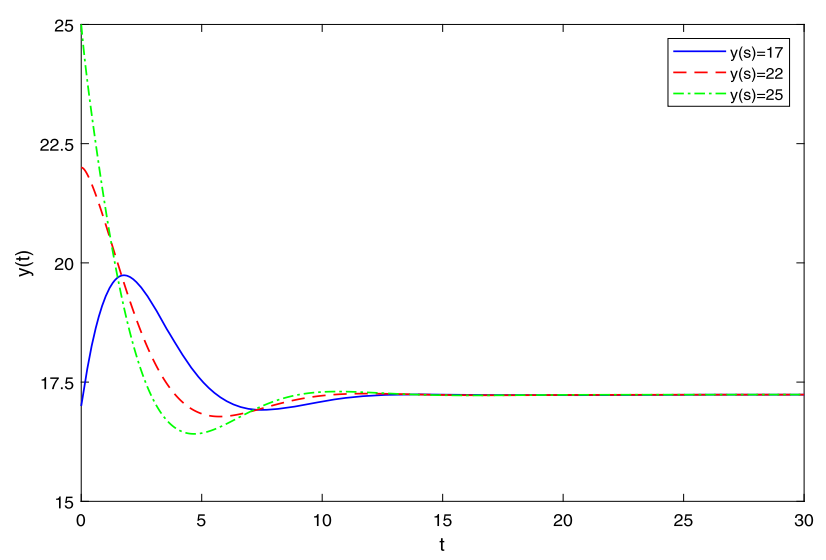

Figure 3 Time responses of state variable $y(t)$ with $\alpha=0.95$

where $\alpha=0.75$, and we take $a=2, a_{1}=0.1, a_{2}=1, b=0.5, b_{1}=1.5, r_{1}=r_{2}=r=0.2, m=1$. By a simple calculation, we have

$$
\left(a_{2}-m r\right)\left(a b-r_{2}\left(b+r_{1}\right)\right)^{2} \approx 0.5917>0.2205=r b_{1}^{2}\left(b+r_{1}\right)^{2} .
$$

It is easy to check that the condition of Theorem 4.1 is satisfied. So, system (5.2) has a unique coexistence equilibrium

$$
\begin{aligned}
& x_{1}^{*}=\frac{a}{b+r_{1}} x_{2}^{*} \approx 1.4286, \quad x_{2}^{*}=\sqrt{\frac{r}{a_{2}-m r}}=0.5, \\
& y^{*}=\frac{a_{2} x_{2}^{*}}{r a_{1}}\left(\frac{a b}{b+r_{1}}-r_{2}-b_{1} x_{2}^{*}\right) \approx 11.9643,
\end{aligned}
$$

which is asymptotically stable. Simulated by Matlab, Figs. 4-6 show the time responses of the variables $x_{1}(t), x_{2}(t)$ and $y(t)$ of system (5.2). 


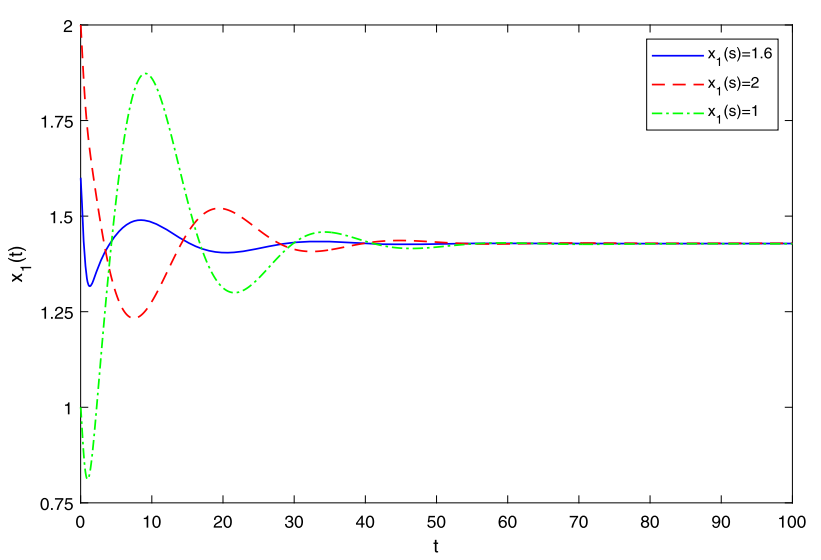

Figure 4 Time responses of state variable $x_{1}(t)$ with $\alpha=0.75$

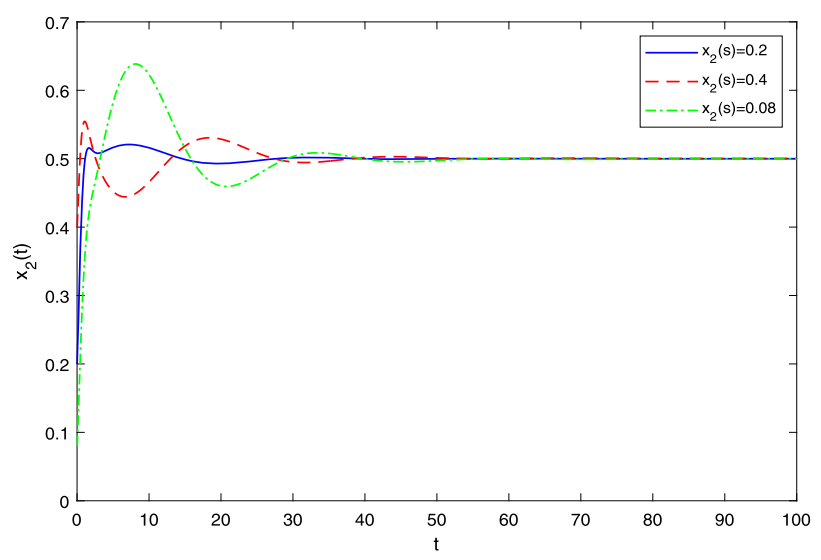

Figure 5 Time responses of state variable $x_{2}(t)$ with $\alpha=0.75$

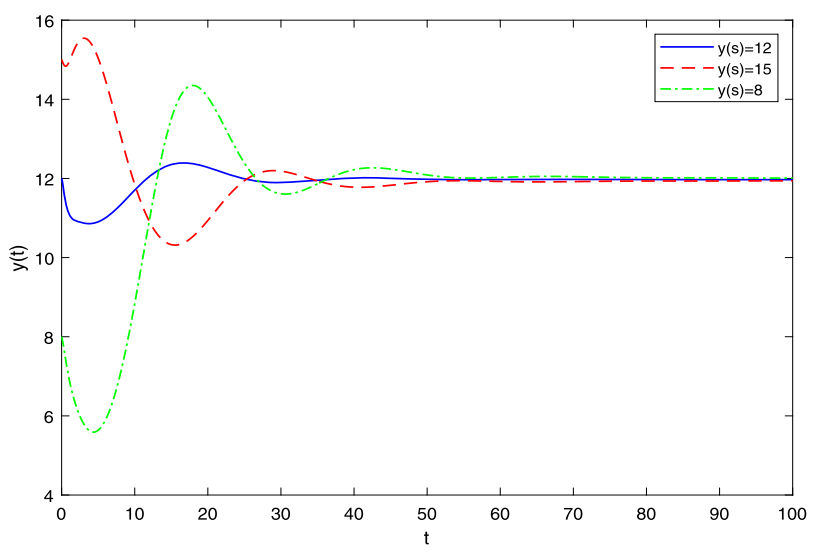

Figure 6 Time responses of state variable $y(t)$ with with $\alpha=0.75$ 


\section{Funding}

This work was supported by the Science Research Fund of Education Department of Yunnan Province of China under Grant 2018JS517.

\section{Availability of data and materials}

Not applicable.

\section{Competing interests}

The authors declare that they have no competing interests.

\section{Authors' contributions}

The authors contributed equally to the writing of this paper. All authors read and approved the final manuscript.

\section{Author details}

${ }^{1}$ School of Mathematics and Statistics, Southwest University, Chongqing 400715, China. ${ }^{2}$ Department of Mathematics and Statistics, Puer University, Puer, Yunnan 665000, China.

\section{Publisher's Note}

Springer Nature remains neutral with regard to jurisdictional claims in published maps and institutional affiliations.

\section{Received: 13 May 2020 Accepted: 7 December 2020 Published online: 28 January 2021}

\section{References}

1. Xu, R., Chaplain, M.A.J., Davidson, F.A.: Permanence and periodicity of a delayed ratio-dependent predator-prey model with stage structure and prey dispersal. Appl. Math. Comput. 159, 823-846 (2004)

2. Wang, L., Li, W.: Periodic and permanence for a delayed nonautonomous ratio-dependent predator-prey model with Holling type functional response. J. Comput. Appl. Math. 162, 341-357 (2004)

3. Huang, Y.J., Chen, F.D., Zhong, L.: Stability analysis of a prey-predator model with Holling type III response function incorporating a prey refuge. Appl. Math. Comput. 182, 672-683 (2006)

4. Georgescu, P., Hsieh, Y.H.: Global dynamics of a predator-prey model with stage structure for the predator. SIAM J. Appl. Math. 67(5), 1379-1395 (2007)

5. Wei, F.Y.: Existence of multiple positive periodic solutions to a periodic predator-prey system with harvesting terms and Holling III type functional response. Commun. Nonlinear Sci. Numer. Simul. 16, 2130-2138 (2011)

6. Banerjee, R., Das, P., Mukherjee, D.: Stability and permanence of a discrete-time two-prey one-predator system with Holling type-III functional response. Chaos Solitons Fractals 117, 240-248 (2018)

7. Dai, Y.F., Zhao, Y., Sang, B.: Four limit cycles in a predator-prey system of Leslie type with generalized Holling type III functional response. Nonlinear Anal., Real World Appl. 50, 218-239 (2019)

8. Li, F., Li, H.: Hopf bifurcation of a predator-prey model with time delay and stage structure for the prey. Math Comput. Model. 55, 672-679 (2012)

9. Peng, M., Zhang, Z.D.: Bifurcation analysis and control of a delayed stage-structured predator-prey model with ratio-dependent Holling type III functional response. J. Vib. Control 26(13-14), 1232-1245 (2020)

10. Miller, K.S., Ross, B.: An Introduction to the Fractional Calculus and Fractional Differential Equations. Wiley, New York (1993)

11. Diethelm, K., Ford, N.J.: Analysis of fractional differential equations. J. Math. Anal. Appl. 265, 229-248 (2002)

12. Jumarie, G.: The Minkowski's space-time is consistent with differential geometry of fractional order. Phys. Lett. A 363 , 5-11 (2007)

13. Ahmed, E., El-Sayed, A.M.A., El-Saka, H.A.A.: Equilibrium points, stability and numerical solutions of fractional-order predator-prey and rabies models. J. Math. Anal. Appl. 325, 542-553 (2007)

14. Jumarie, G.: Table of some basic fractional calculus formulae derived from a modified Riemann-Liouville derivative for non-differentiable functions. Appl. Math. Lett. 22, 378-385 (2009)

15. Deng, J.Q., Deng, Z.M.: Existence of solutions of initial value problems for nonlinear fractional differential equations. Appl. Math. Lett. 32, 6-12 (2014)

16. Choi, S.K., Kang, B., Koo, N.: Stability for Caputo fractional differential systems. Abstr. Appl. Anal. 2014, Article ID $631419(2014)$

17. Wang, F.F., Chen, D.Y., Xu, B.B., Zhang, H.: Nonlinear dynamics of a novel fractional-order Francis hydro-turbine governing system with time delay. Chaos Solitons Fractals 91, 329-338 (2016)

18. Guo, D.D., Yang, G., Feng, X., Han, X.B., Lu, L.G., Ouyang, M.G.: Physics-based fractional-order model with simplified solid phase diffusion of lithium-ion battery. J. Energy Storage 30, 101404 (2020)

19. Shi, L.Y., Yuan, Y., Gao, J.J., Zhou, L., Mao, J.F.: Compact fractional-order model of on-chip inductors with BCB on high resistivity silicon. IEEE Trans. Compon. Packag. Manuf. Technol. 10(5), 878-886 (2020)

20. Huang, C.D., Cai, L.M., Cao, J.D.: Linear control for synchronization of a fractional-order time-delayed chaotic financial system. Chaos Solitons Fractals 113, 326-332 (2018)

21. Xu, C.J., Liao, M.X., Li, P.L., Xiao, Q.M., Yuan, S.: Control strategy for a fractional-order chaotic financial model. Complexity 2019, Article ID 2989204 (2019)

22. Zhang, G.X., Qian, P.X., Su, Z.X.: Evolution of fractional-order chaotic economic systems based on non-degenerate equilibrium points. Chaos Solitons Fractals 128, 219-228 (2019)

23. Faria, A.C.D., Carvalho, A.R.S., Guimarāes, A.R.M., Lopes, A.J., Melo, P.L.: Association of respiratory integer and fractional-order models with structural abnormalities in silicosis. Comput. Methods Programs Biomed. 172, 53-63 (2019)

24. Alavi, M.B., Tabatabaei, M.: Control of depth of anaesthesia using fractional-order adaptive high-gain controller. IET Syst. Biol. 13(1), 36-42 (2018) 
25. Lu, Q., Xiao, M., Cheng, Z.S., Song, Y.R., Huang, C.D., Cao, J.D.: Stability and bifurcation analysis of a fractional-order single-gene regulatory model with delays under a novel $P D^{\alpha}$ control law. Int. J. Biomath. 13(03), 2050016 (2020)

26. Padrón, J.P., Padrón, J.P.P.: Trajectory tracking error using fractional order time-delay recurrent neural networks using Krasovskii-Lur'e functional for Chua's circuit via inverse optimal control. Rev. Mex. Fis. 66(1), 98-104 (2020)

27. Guo, Y.J.: The stability of solutions for a fractional predator-prey system. Abstr. Appl. Anal. 2014, Article ID 124145 (2014)

28. Rihan, F.A., Lakshmanan, S., Hashish, A.H., Rakkiyappan, R., Ahmed, E.: Fractional-order delayed predator-prey systems with Holling type-II functional response. Nonlinear Dyn. 80, 777-789 (2015)

29. Nosrati, K., Shafiee, M.: Dynamic analysis of fractional-order singular Holling type-ll predator-prey system. Appl. Math. Comput. 313, 159-179 (2017)

30. Das, M., Maiti, A., Samanta, G.P.: Stability analysis of a prey-predator fractional order model incorporating prey refuge. Ecol. Genet. Genom. 7-8, 33-46 (2018)

31. Xie, Y.K., Wang, Z., Meng, B., Huang, X.: Dynamical analysis for a fractional-order prey-predator model with Holling III type functional response and discontinuous harvest. Appl. Math. Lett. 106, 106342 (2020)

32. Bhalekar, S., Varsha, D.: A predictor-corrector scheme for solving nonlinear delay differential equations of fractional order. J. Fract. Calc. Appl. 1, 1-9 (2011)

\section{Submit your manuscript to a SpringerOpen ${ }^{\circ}$ journal and benefit from:}

- Convenient online submission

- Rigorous peer review

Open access: articles freely available online

High visibility within the field

- Retaining the copyright to your article

Submit your next manuscript at $\boldsymbol{\nabla}$ springeropen.com 